\title{
Artificial Intelligence in Food Industry
}

\author{
Matthew N. O. Sadiku, Omobayode I. Fagbohungbe, Sarhan M. Musa
}

\author{
Roy G. Perry College of Engineering \\ Prairie View A\&M University \\ Prairie View, TX, USA
}

\begin{abstract}
Artificial Intelligence (AI) is a field of computer science that deals with intelligent machines. AI algorithms, such as machine learning and deep learning, are most commonly used to make intelligent predictions. The application of AI in the food and beverage industry is already transforming the way we think about food production, food manufacturing, food processing, food quality, food delivery, and food consumption. This paper provides an introduction on the applications of AI in food and beverage industry.
\end{abstract}

Key Words: Artificial Intelligence, Food and Beverage Industry, Artificial Intelligence in Food Industry.

\section{INTRODUCTION}

Agriculture, forestry, and fisheries can provide nutritious food for the world. The global food industry continues to grow steadily. (Coronavirus is impacting the global food industry.) The global population is increasing and is widening the gap between food supply and demand. Today, the global population exceeds seven billion. The rising global demand for food can only be met using technology. It also requires developing strong business relationships with farmers and agronomic experts across the globe.

The food industry has always been at the forefront of adopting emerging technologies to improve the sector. Artificial intelligence, with the capacity to makes computers to learn from experience, is playing a predominant role in the food industry. Artificial Intelligence (AI) is poised to revolutionize the food industry. AI is one of emerging technologies that can literally transform the agricultural landscape in the years to come and take agriculture to new heights [1].AI technology can be implemented in all stages of the food supply chain, resulting in an overall improvement and significant increase in efficiency. Forecasting global demands and delivering safe food products can be done using machine learning and deep learning, two of the most commonly used algorithms in the field of AI.

\section{OVERVIEW ON ARTIFICIAL INTELLIGENCE}

Artificial intelligence (AI) is one of the most important global issues of the 21 st century. The modern field of artificial intelligence came into existence in 1956 when the term "artificial intelligence" (AI) was coined by John McCarthy. AI is the branch of computer science that deals with designing intelligent computer systems that mimic human intelligence (e.g. visual perception, speech recognition, decision-making, and language translation). The ability of machines to process natural language, to learn, to plan makes it possible for new tasks to be performed by intelligent systems. The main purpose of AI is to mimic the cognitive function of human beings and perform activities that would typically be performed by a human being. Without being taught by humans, machines use their own experience to solve a problem. Although the original vision for artificial intelligence was to simulate human intelligence, research effort has gradually shifted to autonomous systems that compete with people.

$\mathrm{AI}$ is stand-alone independent electronic entity that functions much like human expert. Today, AI is integrated into our daily lives in several forms, such as personal assistants, automated mass transportation, aviation, computer gaming, facial recognition at passport control, voice recognition on virtual assistants, driverless cars, companion robots, etc. AI technologies are performing better and better at analyzing data [2,3].

An important feature of AI technology is that is can be added to existing technologies. AI has benefited many areas such chemistry and medicine, where routine diagnoses can initiated by AI-aided computers. It embraces a wide range of disciplines such as computer science, engineering, chemistry, biology, physics, astronomy, neuroscience, and social sciences. Figure 1 illustrates the $6 \mathrm{Ws}$ of artificial intelligence [4].

$\mathrm{AI}$ is not a single technology but a range of computational models and algorithms. The major disciplines in $\mathrm{AI}$ include expert systems, fuzzy logic, and artificial neural networks (ANNs), machine learning, deep learning, natural language processing, 
computer vision, and robotics. These computer-based tools or technologies that have been used to achieve AI's goals are illustrated in Figure 2 [5] and explained as follows [6,7]:

- Expert Systems: An expert system (ES) (or knowledge-based system) essentially embodies the knowledge and reasoning of human experts. An ES enables computers to make decisions by interpreting data and selecting between alternatives just as a human expert would do. It uses a technique known as rule-based inference in which rules are used to process data. The main business application of AI is in expert systems, which assist human experts in solving difficult problems.

- $\quad$ Neural Networks: These computer programs identify objects or recognize patterns after having been trained. Artificial neural networks (ANNs) are parallel distributed systems consisting of processing units (neurons) that calculate some mathematical functions. The ANN model represents nonlinear relationships which are directly learned from the data being modeled. Neural networks are being explored for healthcare applications in imaging and diagnoses, risk analysis, lifestyle management and monitoring, health information management, and virtual health assistance.

- Computer Vision: Computer vision comprehends methods and techniques through which artificial vision systems can be constructed. Essentially, a computer vision system is composed of image acquisition and image processing. Computer vision. systems are already widely employed in different segments of agricultural production and industrial food production. They can be used in grading systems for orange, papaya, almond, potato, lemon, wheat, corn, rice, and soybean [8].

- $\quad$ Natural Language Processors: For AI to be useful to us humans, it needs to be able to communicate with us in our language. Computer programs can translate or interpret language as it is spoken by normal people. NLP techniques extract information from unstructured data such as clinical notes to supplement and enrich structured medical data. NLP includes applications such as speech recognition, text analysis, translation and other goals related to language. There are two basic approaches to NLP: statistical and semantic [9]. NLP allows for intelligent search engines, helpful chatbots, and accessibility for people who are visually impaired.

- Robots: These are computer-based programmable machines that have physical manipulators and sensors. Sensors can monitor temperature, humidity, pressure, time, record data, and make critical decisions in some cases. Robots have moved from science fiction to your local hospital. Today, robots perform vital functions in homes, industries, outer space, hospitals, and on military instillations. In jobs with repetitive and monotonous functions they might even completely replace humans. Robotics and autonomous systems are regarded as the fourth industrial revolution. Figure 3 shows a typical use of robots in agriculture [10].

- Fuzzy Logic: Reasoning based on imprecise or incomplete information in terms of a range of values rather than point estimates. Fuzzy logic deals with uncertainty in knowledge that simulates human reasoning in incomplete or fuzzy data. The fuzzy model is robust to parameter changes and tolerant to impression.

- Machine Learning: Algorithms make predictions and interpret data and "learn" without static program instructions. ML is a statistical technique for fitting models to data and training models with data. ML extracts features from input data by constructing analytical data algorithms and examines the features to create predictive models. The most common ML algorithms are supervised learning, unsupervised learning, reinforcement learning, and deep learning. ML algorithms are a good fit for anti-malware solutions because machine learning is well suited to solve "fuzzy" problems. Today, ML models are being developed in the food industry.

- $\quad$ Deep Learning: A subset of machine learning built on a deep hierarchy of layers, with each layer solving different pieces of a complex problem. It aims at increasing the capacity of supervised and unsupervised learning algorithms for solving complex real-world problems by adding multiple processing [11]. The relationship between artificial intelligence, machine learning, and deep learning is shown in Figure 4 [12].

- Data Mining: This deals with the discovery of hidden patterns and new knowledge from large databases. Data mining exhibits a variety of algorithmic tools such as statistics, regression models, neural networks, fuzzy sets, and evolutionary models.

Each AI tool has its own advantages. Using a combination of these models, rather than a single model, is recommended. AI technologies are drastically influencing the retail industry and customer experience. Some types of artificial intelligence are predominant in business, while others are not. AI systems are designed to make decisions using real-time data. They have the ability to learn and adapt as they make decisions. 


\section{AI RESEARCH PROJECTS IN FOOD INDUSTRY}

Several food companies have incorporated machine learning, deep learning into food and beverage products and services. Here are the latest AI research projects related to the food industry [13].

- Predicting food security outcomes

- Food identification

- Ranking food preferences

- Automatic surface area and volume prediction of food

- Generating images of food based on recipes text

- Automatically assign a collective restaurant star rating based on customer reviews of food

- Food recommender system based on the user's history, ingredients, and image of a recipe

- Visual identification of fraudulent foodstuff products

- Food recognition using partially labeled data

- Recipe generation from food images

- Recognizing eating gestures by tracking wrist motion

- Real-time detection of foodborne illness

- Automated food label quality assessments

- Plant seedlings classification

- Automatically assessing the health of individual chickens

- Identification of leaf diseases

\section{APPLICATIONS OF AI IN FOOD INDUSTRY}

In addition to the research projects mentioned above, key areas application of $\mathrm{AI}$ in food industry include production, product development, product customization, marketing, manufacturing, robotics, and processing of food products. AI can also be applied in restaurants, bar and cafe businesses [14-17].

- Agriculture: Agriculture is facing major challenges and AI is regarded more and more as a solution to these challenges. There is an unquestionable growing tendency in the adoption of artificial intelligence in agriculture. Making the right decisions in agricultural activities is less than half the way needed to achieve desired results. The intelligent agents implementing measures into practice are seen more often as a feasible solution for the future. Agriculture automation is the main concern of every country. It has benefited from different automation practices like IoT, wireless communications, machine learning and artificial intelligence, and deep learning. However, the use of technologies in agriculture is still in infancy $[18,19]$.

- Food Processing: Food processing in many places is not fully automated. The food processing industry is benefiting from artificial intelligence, which is helping everything from sorting foods, maintaining health and safety compliances, developing new products, and improving the supply chain. The technology is basically helping to streamline work processes, making the work of employees easier and making operations more efficient. Food processing industry can guarantee complete hygiene and high food quality by automating their processes as much as possible.

- Food Manufacturing: AI can be used by food companies in food manufacturing. Manufacturing of large productions of goods requires big, complicated, and intricately constructed mechanisms. AI in food manufacturing helps to monitor every stage of this process and makes predictions about cost and stock management. Machine learning allows identifying factors affecting the quality and causing flows in the manufacturing process and monitors the path of products from where they are produced and, ultimately, to where customers get it, ensuring transparency.

- Food Production: AI is used in advanced applications to make food production more efficient, safe, and profitable. It has enormous potential to optimize production and uncover manufacturing facilities' best operating points. It can enable faster production changeovers and identifying production bottlenecks before they become a problem. AI can optimize your facility's productivity, efficiency, and output. 
- Food Packaging: AI-powered robotic equipment can accurately perform complex human tasks like the packaging. Robots are meeting the packing and picking demands accelerated by consumers' requests. The complex and laborintensive nature of the process offers unique potential for intelligent automation.

- Food Supply Chain: The food industry needs to supply markets with high-quality food products at reasonable cost to satisfy consumer demands. Supply chains in the food industry are expanding steadily. To enhance food safety, companies can use AI's potential across their supply chain. The technology enables companies to test and monitor food safety products at every step of the supply chain. AI-powered supply chain management systems can be used to monitor and control the activities in the entire supply chain. AI can be used to minimize delays and maximize profit margins by providing close monitoring of every supply chain operation.

- Food Delivery: This is a growing segment of the food industry. The use of AI is a common enabler for most food delivery organizations. Leading food delivery services are using AI to enhance their marketing efforts through automation. AI applications also improve operational efficiency by automating activities like food ordering, dispatching, and billing processes. Food delivery service companies also use AI to enhance their customer services.

- Restaurants: AI is also being used by many apps to recommend the best restaurants based on the location, taste, and previous choices of the user. An AI in food service solution offers to unite the data from various food delivery programs. Self-serving systems are being massively taken up by restaurants as long as they enable customers to control the ordering process.

- Robotics: Robots are widely used in the food industry, but they are only for privileged big food businesses, still unavailable for small-to-medium. Robots are being used from seeding, spraying water and harvesting to cutting, processing and packaging of food products [20]. These are drones to deliver orders or robotic hands that can manage many processes in food manufacturing and even cooking. For example, 7-Eleven claims they use drones in their delivery service. Although robotics is still a quite subtle thing to introduce in some factories, it will bring an obvious benefit in the long run.

Other applications include food safety, food retailers, food market analysis, optical food sorting, predictive maintenance, tracking and traceability, personalized customer service, and heightened consumer engagement.

\section{BENEFITS AND CHALLENGES}

The implementation of $\mathrm{AI}$ in the food and beverage industry is enabling less human errors, less waste of abundant products, save costs, happier customers, optimize and automate processes, and more personalized orders. AI systems deliver more accurate production lines results with greater speed and more consistency than human workers. AI solutions display great potential for the optimization of the hygiene and cleaning tasks, which is the factor number one to influence food safety. Businesses can implement intelligent algorithms to improve their quality of food and services which in turn will mean many healthy meals for the customers [21]. AI could utilize vast datasets of detailed agricultural information for the improvement of our food crops, faster than ever before. Adoption of AI can position companies as digital leaders.

While there are many benefits of using AI in food industry, there are some challenges. AI is yet to be ubiquitous due to cost constraints and lack of skilled experts. The high cost of large-scale deployment in the sector is restricting the market to grow. Integrating new technologies, such as the integration of AI in food companies, is never easy [22]. Besides US and Europe, the adoption of AI is still in the early stages in many nations. AI will never replace humans in the food industry, as humans will always be needed to oversee operations, repair, and maintain old equipment. The technology can essentially work side-by-side with humans to increase operational efficiency.

\section{CONCLUSION}

Artificial Intelligence (AI) is a way of making intelligent machines that think, work, and react like humans. It is the theory and development of computer systems that can perform tasks that normally require human intelligence. AI is pervading and transforming all aspects of the food industry. The implementation of artificial intelligence in the food industry is already moving 
the industry to new heights. While there is much promise in AI, the future depends on deeper engagement among food industry, policy makers, and technologists.

Today, forward-looking businesses in the food industry are already adopting AI to automate different parts of their operations. Tech giants like Google, Microsoft, and IBM are highly interested in developing AI. The entire society can benefit from the use of AI in the food industry. It's time to address world hunger using AI and machine learning. More information on artificial intelligence in the food industry can be found in the following related journals:

- Artificial Intelligence Review

- Artificial Intelligence in Agriculture,

- British Food Journal

- Journal of Food Engineering

- Journal of Agriculture and Food Research

\section{REFERENCES}

[1] M. N. O. Sadiku1, T. J. Ashaolu, and S. M. Musa, "Emerging technologies in agriculture," International Journal of Scientific Advances, vol. 1, no. 1, July-August 2020, pp. 31-34.

[2] M. N. O. Sadiku, T. J. Ashaolu, and S. M. Musa, "Artificial intelligence in medicine: A primer," International Journal of Trend in Research and Development, vol. 6, no. 1, January.-Feb. 2019, pp. 270-272.

[3] Y. Mintz and R. Brodie, "Introduction to artificial intelligence in medicine," Minimally Invasive Therapy \& Allied Technologies, vol. 28, no. 2, 2019, pp. 73-81.

[4] A. Kaplan and M. Haenlein, "Rulers of the world, unite! The challenges and opportunities of artificial intelligence,"

Business Horizons, vol 63, no. 1, January-February 2020, pp. 37-50.

[5] O. Oana, T. Cosmin, and N. C. Valentin, "Artificial intelligence - A new field of computer science which any business should consider," “Ovidius” University Annals, Economic Sciences Series, vol. XVII, no. 1, 2017, pp. 356-360.

[6] R. O. Mason, "Ethical issues in artificial intelligence," Encyclopedia of Information Systems, vol 2, 2003, pp. 239-258.

[7] A. N. Rames et al., "Artificial intelligence in medicine," Annals of the Royal College of Surgeons of England, vol. 86, 2004, pp. 334-338.

[8] D. I. Patrícioa and R. Rieder, "Computer vision and artificial intelligence in precision agriculture for grain crops: A systematic review ," Computers and Electronics in Agriculture, vol. 153, October 2018, pp. 69-81.

[9] M. N. O. Sadiku, Y. Zhou, and S. M. Musa, "Natural language processing in healthcare," International Journal of Advanced Research in Computer Science and Software Engineering, vol. 8, no. 5, May 2018, pp. 39-42.

[10] "4 Agriculture technology projects to transform the industry,"

https://www.ennomotive.com/agriculture-technology-projects/

[11] F. Jiang et al., "Artificial intelligence in healthcare: Past, present and future," Stroke and Vascular Neurology, 2017.

[12] "Quantum computing,"

https://quantumcomputingtech.blogspot.com/2019/05/ai-machine-learning-venn-diagram.html

[13] S. Kurilyak, "Coronavirus update: Food and artificial intelligence," February 2019,

https://blog.produvia.com/artificial-intelligence-ai-in-food-industry-ec8e925fa35e

[14] K. Utermohlen, "4 Applications of artificial intelligence in the food industry," January 2019,

https://heartbeat.fritz.ai/4-applications-of-artificial-intelligence-ai-in-the-food-industry-

e742d7c02948\#: :text=1)\%20Sorting\%20Food,of\%20canned\%20and\%20bagged\%20goods.\&text=One\%20of\%20the\%20most $\%$

20advanced,solutions\%20with\%20machine\%20learning\%20functionalities.

[15] "Machine learning and artificial intelligence in the food industry," August 2019,

https://medium.com/@spd.group/machine-learning-and-artificial-intelligence-in-the-food-industry-598f78471106

[16] "How artificial intelligence is revolutionizing the food and beverage industry," May 2020,

https://new.abb.com/news/detail/61772/how-artificial-intelligence-is-revolutionizing-the-food-and-beverage-

industry\#: :text=AI\%20can\%20play\%20a\%20vital,complexity\%20to\%20the\%20production\%20line.

[17] "Applications of artificial intelligence (AI) in the food industry,"

https://morioh.com/p/6f346ea78169

[18] " Adoption of artificial intelligence in agriculture," Bulletin UASVM Agriculture, vol. 68, no. 1, 2011, pp. 284-293.

[19] K. Jha et al., "A comprehensive review on automation in agriculture using artificial intelligence," Artificial Intelligence in Agriculture, vol 2, June 2019, pp. 1-12.

[20] J. Iqbal, Z. H. Khan, and A. Khalid, "Prospects of robotics in food industry,"

Food Science and Technology, viol. 37, no. 2, April-June 2017, pp. 159-165. 
[21] A. Eliazàt, “4 Ways AI is revolutionizing the food industry,” June 2020, https://autome.me/4-ways-ai-is-revolutionizing-the-food-industry/

[22] "Artificial intelligence applications in food industry," October 2019, https://www.linkedin.com/pulse/artificialintelligence-applications-food-industry-mert-damlapinar

\section{ABOUT THE AUTHORS}

Matthew N.O. Sadiku is a professor in the Department of Electrical and Computer Engineering at Prairie View A\&M University, Prairie View, Texas. He is the author of several books and papers. His areas of research interests include computational electromagnetics and computer networks. He is a fellow of IEEE.

Omobayode I. Fagbohungbe is a doctoral student at Prairie View A\&M University, Prairie View Texas. He holds a masters of science degree in control engineering from the University of Manchester, UK and a bachelor degree in electrical and electronics engineering from Obafemi Awolowo University, Nigeria. He is a graduate member of the IEEE. His current research interests are in data science, machine learning, and deep learning.

Sarhan M. Musa is a professor in the Department of Electrical and Computer Engineering at Prairie View A\&M University, Texas. He has been the director of Prairie View Networking Academy, Texas, since 2004. He is an LTD Sprint and Boeing Welliver Fellow. His research interests include computer networks and computational electromagnetics.

\begin{tabular}{|l|l|l|l}
\hline WHY & $\begin{array}{l}\text { Misconceptions exist because media tend to emphasize the dangers and threats of Al } \\
\text { more than its potential and opportunities }\end{array}$ \\
\hline WHAT & $\begin{array}{l}\text { Al is "a system's ability to interpret external data correctly, to learn from such data } \\
\text { and to use those learnings to achieve specific goals and tasks through flexible } \\
\text { adaptation" (Kaplan \& Haenlein, 2019b, p. 17) }\end{array}$ \\
\hline WHO & $\begin{array}{l}\text { As with any tool, Al-driven robots can be used for good or evil. It is unclear if robots can } \\
\text { turn evil by themselves (wireheading), but likely that they do not and will not think } \\
\text { like humans }\end{array}$ \\
\hline WHEN & $\begin{array}{l}\text { It is impossible to say when artificial superintelligence will arrive- maybe tomorrow, in } \\
\text { our lifetime, or never }\end{array}$ \\
\hline WHERE & $\begin{array}{l}\text { The current epicenters of Al development are China and the U.S., but other world } \\
\text { regions (especially Europe) will play important (niche) roles in the fugure }\end{array}$ \\
\hline HOW & $\begin{array}{l}\text { The extent of Al risk is hard to predict but likely to be substantial. Even if the chance } \\
\text { of occurrence is small, the discounted risk is sufficiently large to warrant preventive } \\
\text { measures today }\end{array}$ \\
\hline
\end{tabular}

Figure 1 The 6 Ws of artificial intelligence [4]. 
International Journal of Engineering Research And Advanced Technology, Vol.6 (10), October-2020

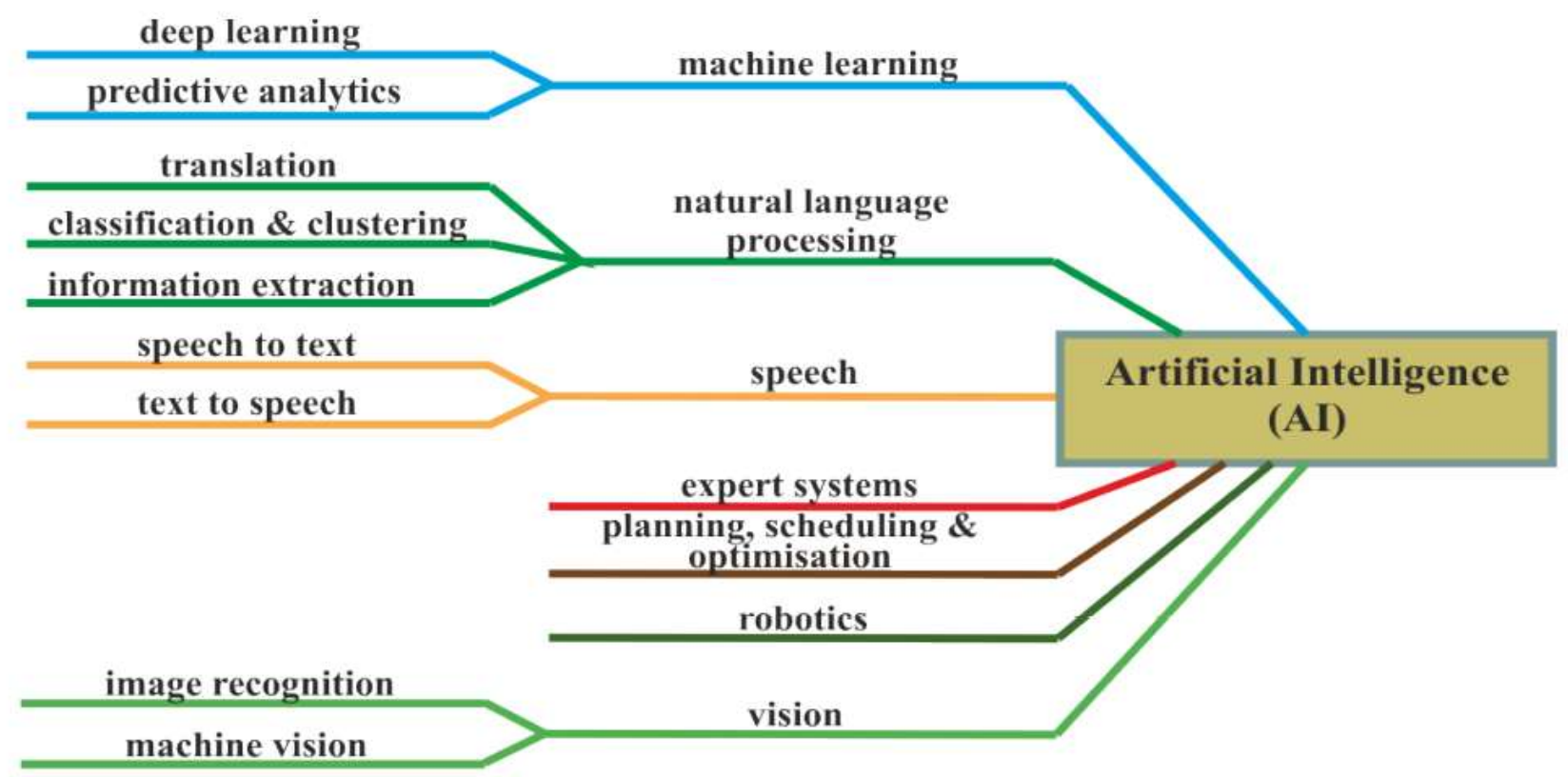

Figure 2 Many branches of artificial intelligence [5].

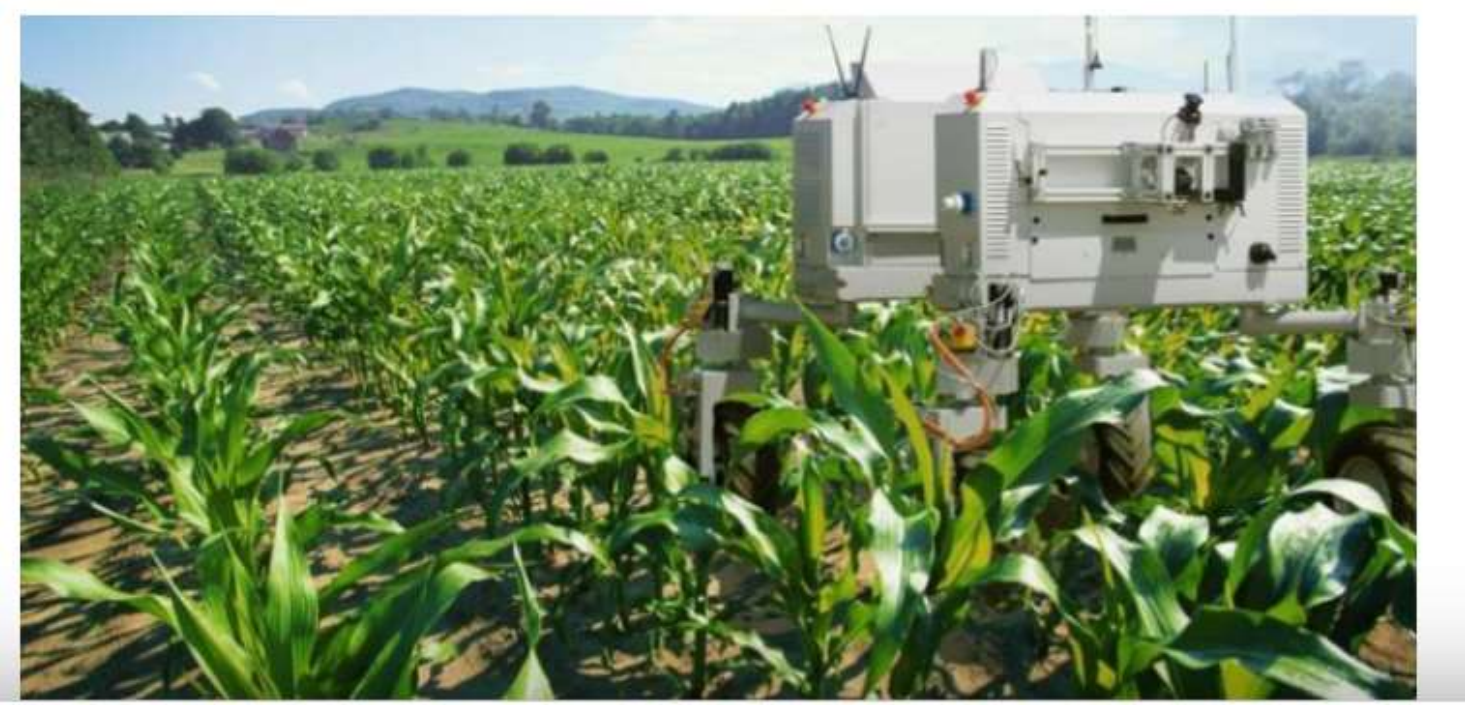

Figure 3 Use of robots in agriculture [10]. 
International Journal of Engineering Research And Advanced Technology, Vol.6 (10), October-2020

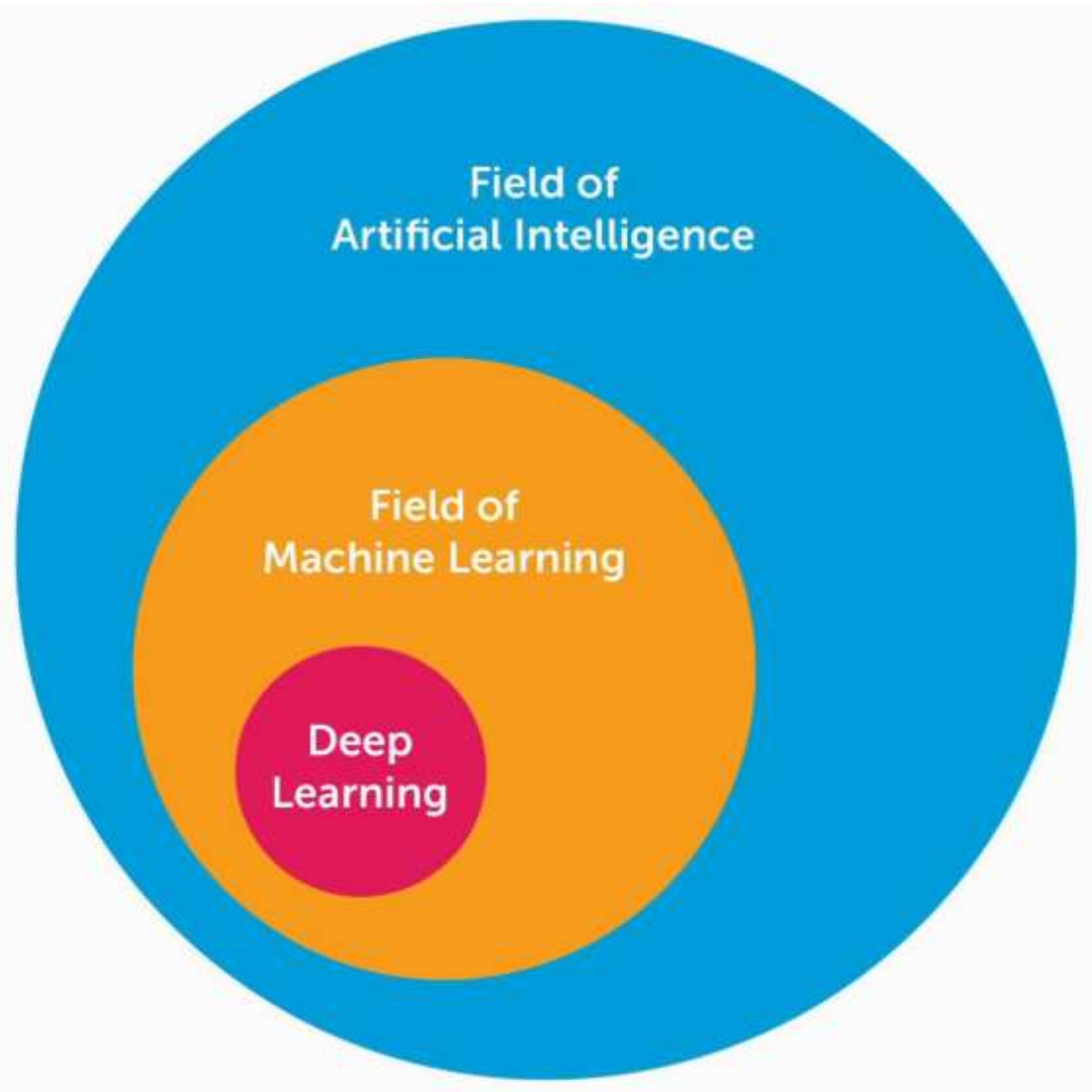

Figure 4 The relationship between AI, machine learning, and deep learning [12]. 